\title{
Transconductance of a double quantum dot system in the kondo regime
}

Koerting, V.; Wofle, P.; Paaske, Jens

Published in:

Physical Review Letters

DOI:

10.1103/PhysRevLett.99.036807

Publication date:

2007

Document version

Early version, also known as pre-print

Citation for published version (APA):

Koerting, V., Wofle, P., \& Paaske, J. (2007). Transconductance of a double quantum dot system in the kondo regime. Physical Review Letters, 99(3), 036807. https://doi.org/10.1103/PhysRevLett.99.036807 


\title{
Transconductance of a Double Quantum Dot System in the Kondo Regime
}

\author{
V. Koerting* and P. Wölfle \\ Institut für Theorie der Kondensierten Materie, Universität Karlsruhe, D-76128 Karlsruhe, Germany
}

\section{J. Paaske}

The Niels Bohr Institute and Nano-Science Center, University of Copenhagen, DK-2100 Copenhagen, Denmark (Received 21 December 2006; published 20 July 2007)

\begin{abstract}
We consider a lateral double-dot system in the Coulomb blockade regime with a single spin-1/2 on each dot, mutually coupled by an antiferromagnetic exchange interaction. Each of the two dots is contacted by two leads. We demonstrate that the voltage across one of the dots will have a profound influence on the current passing through the other dot. Using poor man's scaling, we find that the Kondo effect can lead to a strong enhancement of this transconductance.
\end{abstract}

Nanostructured electron systems offer the unique possibility to prepare and probe highly nonequilibrium stationary states. Energy dissipation and therefore heating, characteristic of any nonequilibrium state, take place in the bulk reservoirs connected to the nanostructure, where it is absorbed causing a negligible change of state. The nature of such a nonequilibrium state may be conveniently probed by transport measurements rather than thermodynamic or optical experiments (cf., e.g., Refs. [1,2]). In an ordinary quantum dot setup the probing field, e.g., the applied bias voltage, is also responsible for driving the system out of equilibrium. However, by separating the driving field and the probing field many more possibilities for both studying nonequilibrium states and exploiting their properties arise. In the simplest realization of such a system one must have two parts of the system strongly coupled by some interaction and each contacted by two leads. Applying a sufficiently high bias across one part will then influence the current through the other part. This coupling is mediated by the change in nonequilibrium occupation numbers on the nanostructure caused by the driving field, which in turn governs the current response to the probing field.

In this Letter we investigate the properties of a system of this type (cf. Fig. 1), that is two quantum dots in the Coulomb blockade regime with odd electron occupation, giving rise to spins $S_{L, R}=1 / 2$, coupled by an exchange interaction $K$. Each dot is contacted by two leads, modeled by exchange-tunneling amplitudes $J_{L, R} \sim t^{2} / E_{C}$, in terms of lead-dot tunneling amplitudes $t$ and charging energy $E_{C}$. This setup is very similar to the double-dot devices studied, for example, in Refs. [3-5], where the central region was either a quantum dot, a quantum wire, or a tunneling barrier, which could then support an exchange interaction between the two spins. The following discussion does not rely on the physical nature of the exchange coupling. In practice, it can be due to a simple superexchange mechanism [6] or a RKKY interaction, as is most likely the case in Refs. [3,4], or even due to the so-called Kondo-doublet interaction suggested recently [7].
The theory of the two-impurity Kondo model per se is well established [8], but with the experimental progress in quantum dot systems, issues like the quantum critical properties remain an active field of research [9-11]. The influence of the exchange interaction $K$ and an applied magnetic field on the nonlinear conductance $d I_{L} / d V_{L}$ was studied in Refs. [12,13], and it was demonstrated how the nature of the ground state (Kondo screened or local singlet) can be extracted from the temperature dependence of the linear conductance.

In the present setup with two sets of biased leads, we are facing an entirely new problem in which correlations and nonequilibrium effects must be treated side by side. We calculate the transconductance $d I_{L} / d V_{R}$ and demonstrate a transistor effect in which a current through the left dot $I_{L}$ as a response to a bias on the left dot $V_{L}$ is switched on only when the bias over the right $\operatorname{dot} V_{R}$ exceeds a threshold given by $K>0$. This threshold effect rests on the fact that the exchange-tunneling current from lead 1 to lead $2, I_{L}$, is zero as long as the spins are locked in a singlet. A sufficiently large bias across the right dot will, however, lead to a partial occupation of the triplet state, which does allow for a finite current $I_{L}$. As we shall demonstrate, the nonequilibrium polarization due to $V_{R}$, and thereby the trans-
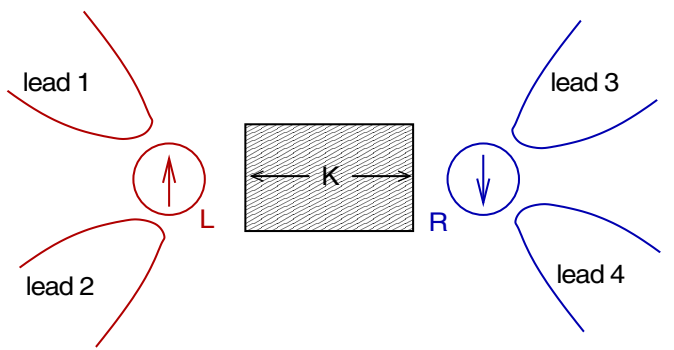

FIG. 1 (color online). Double quantum dot system: Two Kondo impurities denoted $L, R$ each connected to two leads 1 , 2 and 3, 4, respectively, and coupled to each other by a spin exchange interaction $K$. 
conductance peak at $V_{R} \sim K$, will be enhanced by the Kondo effect. In the case of ferromagnetic coupling, the triplet ground state gives rise to a spin-1 Kondo effect $[12,13]$. In this case, a bias voltage over the right quantum dot will influence the corresponding zero-bias conductance peak and singlet satellite peaks in $d I_{L} / d V_{L}$, partly by decoherence and partly by a redistribution of the singlet or triplet occupations. However, these effects are less dramatic and we therefore restrict our attention to the antiferromagnetic case, $K>0$, which exhibits the aforementioned threshold effect.

Model. - We model the double dot by two spin- $\frac{1}{2}$ operators $\mathbf{S}_{L}, \mathbf{S}_{R}$ mutually coupled by exchange interaction $K$ and each coupled to a pair of leads (couplings $J_{L, R}>0$ ) as sketched in Fig. 1. The Hamiltonian reads

$$
\begin{aligned}
H= & \sum_{\mathbf{k} n \sigma}\left(\epsilon_{k}-\mu_{n}\right) c_{n \mathbf{k} \sigma}^{\dagger} c_{n \mathbf{k} \sigma}+K \mathbf{S}_{L} \cdot \mathbf{S}_{R} \\
& +\sum_{n, m=1,2} J_{L}^{n m} \mathbf{S}_{L} \cdot \mathbf{s}_{n m}+\sum_{n, m=3,4} J_{R}^{n m} \mathbf{S}_{R} \cdot \mathbf{s}_{n m},
\end{aligned}
$$

where $n, m=1,2,3,4$ label the leads, assumed to be in equilibrium at chemical potentials $\mu_{n} . c_{n \mathbf{k} \sigma}^{\dagger}$ creates electrons in lead $n$ of momentum $\mathbf{k}$ and spin $\sigma$ and the spin operator $\mathbf{s}_{n m}=\sum_{\mathbf{k}, \mathbf{k}^{\prime}, \sigma, \sigma^{\prime}} c_{n \mathbf{k} \sigma}^{\dagger}\left(\vec{\tau}_{\sigma, \sigma /} / 2\right) c_{m \mathbf{k}^{\prime} \sigma^{\prime}}$ denotes, respectively, the conduction electron spin $(n=m)$ and the exchange-tunneling operator $(n \neq m)$. We do not allow for charge transfer between the two dots. Experimentally the charge transfer may be suppressed by a suitable arrangement of tunneling barriers (cf. [9]).

For $K=0$, the two spins will undergo separate screening by the conduction electron spins in each set of leads as the temperature is lowered beneath the two, generally different, Kondo temperatures $T_{K, L}$ and $T_{K, R}$. However, at sufficiently large bias voltages $e V_{L} \gg T_{K, L}$ and/or $e V_{R} \gg$ $T_{K, R}$, where $\mathrm{eV}_{L}, \mathrm{eV}_{R}$ are the chemical potential differences of leads $n=1,2$ or leads $n=3$, 4, respectively, these Kondo effects are partially suppressed [14,15]. A finite antiferromagnetic exchange coupling $K$, on the other hand, will correlate the spins $\mathbf{S}_{L}, \mathbf{S}_{R}$ and, depending on the relative magnitude of $K$ and the Kondo temperatures, the two spins will either lock into a singlet or be screened by their respective lead electrons [8]. We will be interested in the regime $K \gg \max \left\{T_{K, L}, T_{K, R}\right\}$, when the Kondo effect cannot develop fully. In this case it is necessary to treat the exchange coupling $K$ exactly, whereas it is possible to treat the Kondo effect perturbatively.

The spin states of the isolated double dot are the singlet $(S=0)$ and triplet $(S=1)$ states of the two coupled spin-1/2. They form the appropriate basis states in this regime even when the coupling to the leads is turned on. For convenience, we employ a pseudoboson (bond operator [16]) representation of the four impurity states, with creation operators $\left\{b_{\gamma}^{\dagger}\right\}=\left(s^{\dagger}, t_{+}^{\dagger}, t_{0}^{\dagger}, t_{-}^{\dagger}\right)$, i.e., $\gamma \in$ $\{s,+, 0,-\}$. The energy eigenvalues of the four states are $\omega_{s}=-\frac{3}{4} K, \omega_{0}=\omega_{+,-}=\frac{1}{4} K=\omega_{t}$, and therefore
$K \mathbf{S}_{L} \cdot \mathbf{S}_{R}=\sum_{\gamma} \omega_{\gamma} b_{\gamma}^{\dagger} b_{\gamma}$, subjected to the constraint $Q=$ $s^{\dagger} s+t_{0}^{\dagger} t_{0}+t_{+}^{\dagger} t_{+}+t_{-}^{\dagger} t_{-}=1$. The constraint may be exactly imposed by adding a term $\lambda Q$ to the Hamiltonian and taking the limit $\lambda \rightarrow \infty$ [17]. In terms of the pseudobosons, the spin- $1 / 2$ operators are given by $S_{L, R}^{z}=\frac{1}{2}\left( \pm s^{\dagger} t_{0} \pm t_{0}^{\dagger} s+t_{+}^{\dagger} t_{+}-t_{-}^{\dagger} t_{-}\right) \quad$ and $\quad S_{L, R}^{+}=$ $\frac{1}{\sqrt{2}}\left(S_{L, R}^{x}+i S_{L, R}^{y}\right)=\left(S_{L, R}^{-}\right)^{\dagger}=\frac{1}{2}\left( \pm s^{\dagger} t_{-} \mp t_{+}^{\dagger} s+t_{+}^{\dagger} t_{0}+t_{0}^{\dagger} t_{-}\right)$ [16], or in compact notation $\mathbf{S}_{L, R}=\frac{1}{2} \sum_{\gamma, \gamma^{\prime}} b_{\gamma}^{\dagger} \mathbf{T}_{L, R ; \gamma \gamma^{\prime}} b_{\gamma^{\prime}}$, where $\mathbf{T}_{L, R ; \gamma \gamma^{\prime}}$ is a vector of three $4 \times 4$ matrices $T^{x}, T^{y}, T^{z}$. Notice that this exchange-tunneling model contains no operators of the form $s^{\dagger} s$, which immediately implies that the current $I_{L}$ is zero unless transitions to the triplet states become energetically allowed.

It is known that away from the particle-hole symmetric point a finite potential scattering term will give rise to a current below the threshold. Nevertheless, we shall assume that a set of individual gates on each dot can be adjusted so as to make the system (nearly) particle-hole symmetric and hence neglect potential scattering altogether. In the setup by Sasaki et al. [4], such tuning was made possible by two individual plunger gates.

Nonequilibrium perturbation theory.-In equilibrium, i.e., for vanishing bias voltages, the states $|\gamma\rangle$ of the double dot are thermally occupied, with occupation numbers $n_{\gamma}=$ $\left\langle b_{\gamma}^{\dagger} b_{\gamma}\right\rangle=\exp \left(-\omega_{\gamma} / T\right) / \sum_{\gamma^{\prime}} \exp \left(-\omega_{\gamma^{\prime}} / T\right)$. In a stationary nonequilibrium situation with sufficiently large currents through the nanostructure, the occupation numbers are determined by the currents, i.e., the couplings and the voltages, rather than temperature [18]. A further complication is that nondiagonal parts of the density matrix $n_{\gamma \gamma^{\prime}}=\left\langle b_{\gamma}^{\dagger} b_{\gamma^{\prime}}\right\rangle$ may be nonzero due to the mixing of states by the coupling to the leads. Confining our considerations to zero magnetic field this will not be the case and, furthermore, the triplet states will be equally occupied, i.e., $n_{\gamma}=n_{t}$, for $\gamma=+, 0$, - . It is useful to define the polarization $p=n_{s}-n_{t}$, and we note that $\left\langle\mathbf{S}_{L} \cdot \mathbf{S}_{R}\right\rangle=-\frac{3}{4} p$.

The nonequilibrium occupation numbers are determined from the steady-state quantum Boltzmann equation, $\sum_{\gamma^{\prime} \neq \gamma}\left[\Gamma_{\gamma \gamma^{\prime}} n_{\gamma^{\prime}}-\Gamma_{\gamma^{\prime} \gamma} n_{\gamma}\right]=0$, with transition rates given by the golden rule expression

$$
\begin{gathered}
\Gamma_{\gamma \gamma^{\prime}}=\frac{\pi}{4} \sum_{n, m} \mathbf{T}_{\gamma^{\prime} \gamma}^{m n} \cdot \mathbf{T}_{\gamma \gamma^{\prime}}^{n m} \int_{-\infty}^{\infty} d \omega g_{\gamma \gamma^{\prime}}^{n m}(\omega) g_{\gamma^{\prime} \gamma}^{m n}\left(\omega-\omega_{\gamma^{\prime} \gamma}\right) \\
\times F_{\gamma \gamma^{\prime}}^{n m}(\omega),
\end{gathered}
$$

where $(n, m)$ takes the values $(1,2)$ or $(3,4)$ and $\mathbf{T}^{n m}=\mathbf{T}_{L}$ or $\mathbf{T}_{R}, \omega_{\gamma \gamma^{\prime}}=\omega_{\gamma}-\omega_{\gamma^{\prime}}$, and $F_{\gamma \gamma^{\prime}}^{n m}(\omega)=f\left(\omega-\mu_{n}\right)[1-$ $\left.f\left(\omega-\mu_{m}-\omega_{\gamma \gamma^{\prime}}\right)\right], f(\omega)$ being the Fermi function. The dimensionless exchange couplings, $g^{n m}=\nu_{F} J^{n m}$ (assuming same density of states $\nu_{F}$ in all leads), will only acquire frequency dependence under renormalization as we shall discuss later. The Boltzmann equation is readily solved together with the constraint $\sum_{\gamma} n_{\gamma}=1$, and using the threefold degeneracy of the triplet, one finds that $p=$ $\left(\Gamma_{s t}-\Gamma_{t s}\right) /\left(\Gamma_{s t}+3 \Gamma_{t s}\right)$. 
The current through the left dot is obtained as

$$
\begin{aligned}
I_{L}(V)= & \frac{\pi^{2}}{2} \frac{e}{h} \sum_{\gamma \gamma^{\prime}} n_{\gamma} \mathbf{T}_{L ; \gamma \gamma^{\prime}} \cdot \mathbf{T}_{L ; \gamma^{\prime} \gamma} \int_{-\infty}^{\infty} d \omega g_{\gamma \gamma^{\prime}}^{21}(\omega) \\
& \times g_{\gamma^{\prime} \gamma}^{12}\left(\omega-\omega_{\gamma^{\prime} \gamma}\right)\left[F_{\gamma^{\prime} \gamma}^{12}(\omega)-F_{\gamma^{\prime} \gamma}^{21}(\omega)\right],
\end{aligned}
$$

and a corresponding expression holds for $I_{R}(V)$, with leads 1,2 replaced by 3,4 .

From Eq. (3) it is clear that $V_{R}$ only enters via the occupation numbers, and using the unrenormalized couplings the transconductance is given by

$$
\frac{d I_{L}}{d V_{R}}=\frac{6 \pi^{2} e}{h K} g_{c}^{2} p^{2}\left[F_{-}\left(V_{L}\right)-e V_{L}\right] \frac{d}{d V_{R}} F_{+}\left(V_{R}\right),
$$

where $F_{ \pm}(V)=(K-e V) n_{B}(K-e V) \pm(K+e V) n_{B}(K+e V)$, in terms of the Bose function $n_{B}(\varepsilon)=1 /\left(e^{\varepsilon / T}-1\right)$, and $g_{c}^{2}=\left(g^{12} g^{34}\right)^{2} / \sum_{m, n}\left(g^{m n}\right)^{2} \cdot d I_{L} / d V_{R}$ is an odd function of $V_{R}$ which diverges like $T /\left(K-e V_{R}\right)$ for $e V_{R} \rightarrow K$. As we shall see below, this divergence is removed once the broadening of the excited triplet states is taken into account, and one is left with a pronounced peak in the transconductance. Assuming that $g^{m n}=g_{L(R)}$ for $m, n \in\{1,2\},\{3,4\}$, one has $g_{c}^{2}=\left(g_{L}^{-2}+g_{R}^{-2}\right)^{-1}$ which shows that the transconductance peak is expected to be largest in a symmetric setup where $g_{L}=g_{R}$.

Renormalized perturbation theory. - Since the higher order terms in the perturbation series are plagued by logarithmic singularities characteristic of the Kondo effect, we apply poor man's scaling to sum up the leading logarithms in this series. As demonstrated earlier $[15,19]$, this is conveniently done by keeping track of the generated frequency dependence of the couplings. Within this approach the renormalized coupling functions are calculated from the following set of renormalization group (RG) equations describing the flow of couplings under a reduction of the bandwidth $D$ :

$$
\begin{aligned}
\frac{\partial g_{\mathrm{tt}}^{n m}(\omega)}{\partial \ln D}= & -\frac{1}{2} \sum_{l}\left\{g_{s t}^{l m}\left(\mu_{l}\right) g_{t s}^{n l}\left(\mu_{l}-K\right) \Theta_{\omega-\mu_{l}+K}\right. \\
& +g_{s t}^{n l}\left(\mu_{l}+K\right) g_{t s}^{l m}\left(\mu_{l}\right) \Theta_{\omega-\mu_{l}-K} \\
& \left.+2 g_{\mathrm{tt}}^{l m}\left(\mu_{l}\right) g_{\mathrm{tt}}^{n l}\left(\mu_{l}\right) \Theta_{\omega-\mu_{l}}\right\}, \\
\frac{\partial g_{t s}^{n m}(\omega)}{\partial \ln D}= & -\sum_{l}\left\{g_{t s}^{l m}\left(\mu_{l}\right) g_{\mathrm{tt}}^{n l}\left(\mu_{l}\right) \Theta_{\omega-\mu_{l}}\right. \\
& \left.+g_{\mathrm{ts}}^{n l}\left(\mu_{l}-K\right) g_{\mathrm{tt}}^{l m}\left(\mu_{l}\right) \Theta_{\omega-\mu_{l}+K}\right\},
\end{aligned}
$$

with $g_{s t}^{n m}(\omega)=g_{t s}^{m n}(\omega-K)$. Here $\Theta_{\omega}=\Theta\left(D^{2}-\omega^{2}-\right.$ $\Gamma^{2}$ ) is the step function and $\Gamma$ is a Korringa-like decoherence rate due to particle-hole excitations in the leads. In general, $\Gamma$ involves both self-energy and vertex corrections and may depend on the intermediate state for which it describes the broadening (cf. [20]). Since, however, $\Gamma$ appears only under the logarithm we will neglect these details here and simply use the maximum $\Gamma=\max \left\{\Gamma_{\gamma \gamma^{\prime}}\right\}$ with $\Gamma_{\gamma \gamma^{\prime}}$ defined in Eq. (2). The renormalized couplings and $\Gamma$ are calculated self-consistently, then we solve for the occupation numbers and calculate the current using the renormalized coupling functions in Eq. (3). The perturbative RG approach is valid for $K \gg T_{K}$, where $T_{K}=$ $D_{0} e^{-1 / 2 g\left(D_{0}\right)}$ in terms of bare coupling $g\left(D_{0}\right)$ and the half-bandwidth $D_{0}$.

Renormalized conductances. - In Fig. 2 we plot the polarization $p$ as a function of $e V_{R} / K$ at $V_{L}=0$, for several values of $K / T_{K}$ and at temperature $T / T_{K}=0.1$. As $e V_{R}$ approaches $K$ the triplet states become partially populated and eventually $p$ tends to zero as $1 / V_{R}$. Since we use $T \ll K$, temperature is practically zero and the broadening is governed by $K$ and $V_{R}$. In the inset of Fig. 2 we plot the derivative $-K d p / d V_{R}$ vs $e V_{R} / K$, showing a pronounced peak which is increasingly smeared as the ratio $K / T_{K}$ is made smaller and the Kondo correlations become more pronounced.

From the polarization $p$ and the renormalized couplings, we can now determine the current from Eq. (3). As mentioned above, electron transport via exchange tunneling always involves excitation out of the singlet ground state. This requires an energy of the order of $K$ and therefore leads to a threshold behavior near $e V_{L} \ll K$, as sketched also in Ref. [12]. Alternatively, the singlet-triplet gap can be overcome by a finite $V_{R}$, which will lead to a finite $d I_{L} / d V_{L}$ even for $e V_{L}<K$. This is clearly seen in Fig. 3. When $V_{R}$ becomes larger than $K$, a zero-bias peak is seen to develop, the magnitude of which is determined by the ratio $\Gamma K / T_{K}^{2}$. In the limit of zero probing voltage, we find that

$$
\lim _{V_{L} \rightarrow 0} \frac{d I_{L}}{d V_{L}}=\frac{6 \pi^{2} e^{2}}{h} \frac{n_{t}}{\left[4 \ln \left(\sqrt{\Gamma K} / T_{K}\right)\right]^{2}},
$$

where both $n_{t}$ and $\Gamma$ depend on the driving-voltage $V_{R}$. The inset of Fig. 3 shows the increase of the linear conductance with $V_{R}$, as calculated, respectively, by perturbative RG and second order perturbation theory (PT). The initial

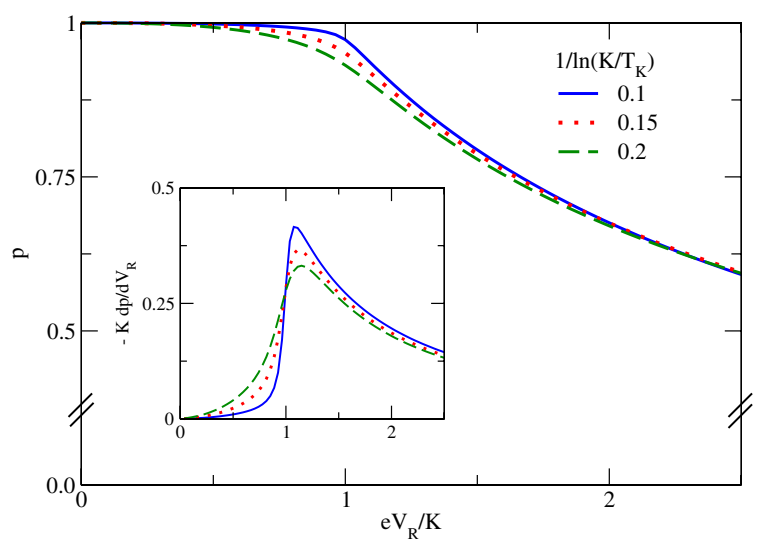

FIG. 2 (color online). Polarization $p=n_{s}-n_{t}$ versus voltage $e V_{R} / K$ for different values of $K / T_{K}$ at $T / T_{K}=0.1$ and voltage $V_{L}=0$. Inset shows the derivative of the curves in the main panel. 


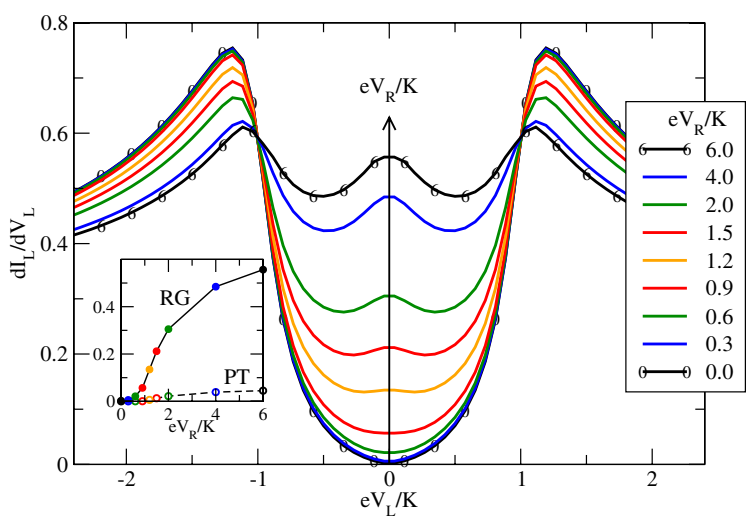

FIG. 3 (color online). Differential conductance $d I_{L} / d V_{L}$ in units of $e^{2} / h$ for fixed $T / T_{K}=0.1$ and $1 / \ln \left(K / T_{K}\right)=0.2$ versus $e V_{L} / K$ at different values of $e V_{R} / K$. Inset: $d I_{L} / d V_{L}$ at $V_{L} \rightarrow 0$ versus $e V_{R} / K$. Solid circles: perturbative RG; open circles: second order PT.

rise with $V_{R}$ is set by the triplet occupation number $n_{t}=$ $(1-p) / 4$. The amplitude is logarithmically enhanced due to the Kondo effect and falls off very slowly $\left(\Gamma \propto V_{R}\right)$ for $V_{R} \gg K$.

Figure 4 shows the transconductance versus $e V_{R} / K$ for $V_{L} \rightarrow 0$. Enhancing the Kondo correlations by lowering $K / T_{K}$ is seen to amplify the transconductance but also to smear the threshold. By scaling with $K / e V_{L}$ we eliminate the $1 / K$ dependence seen in Eq. (4) as well as most of the dependence on the probing field for $e V_{L}<K$ (cf. inset of Fig. 4).

Conclusion. - We studied the mutual influence of spatially separated currents flowing through two quantum dots coupled only by spin exchange. The strong interrelation between the currents and nonequilibrium occupation numbers for the singlet and triplet spin states correlates the two currents, and we showed that this can be observed as a

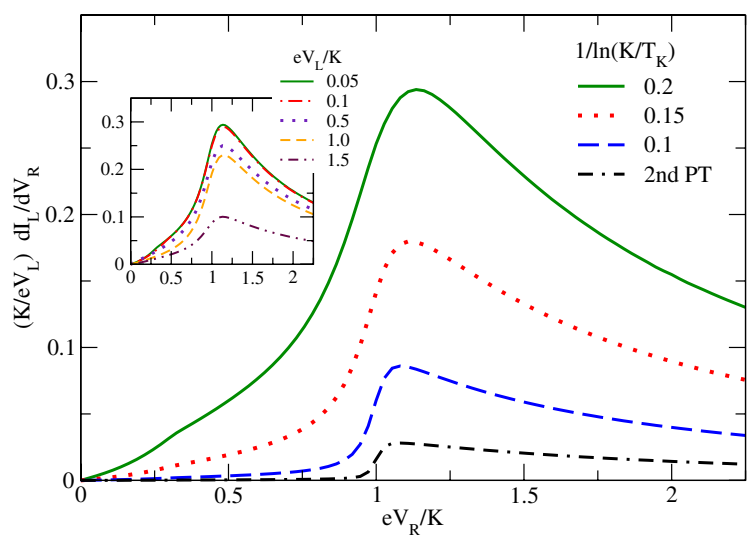

FIG. 4 (color online). Transconductance $d I_{L} / d V_{R}$ in units of $e^{2} / h$ multiplied by $\left(K / e V_{L}\right)$ versus $e V_{R} / K$ for fixed $T / T_{K}=$ 0.1 , probing voltage $e V_{L} / K=0.05$, and different values of $K / T_{K}$. Inset: Transconductance for $1 / \ln \left(K / T_{K}\right)=0.2$ and different $e V_{L} / K$. marked peak in the transconductance. Since the spin exchange interaction requires well-defined spins on the quantum dots, the coupling to the leads should be relatively small, but opening up towards the Kondo regime our perturbative RG calculations demonstrate that weak Kondo correlations $\left(T_{K} \ll K\right)$ will in fact enhance the transconductance peak. This pronounced signal in the transconductance provides a new experimental means of probing the exchange coupling between two quantum dot spins. Moreover, as evident from Eq. (7), this mode of operation provides direct experimental evidence for the voltage dependence of the nonequilibrium distribution function $n_{t}$.

We acknowledge discussions with Chung-Hou Chung, Lars Fritz, and Matthias Vojta. This work has been supported by the DFG-Center for Functional Nanostructures (CFN) at the University of Karlsruhe under project B2.9 (V. K. and P.W.), the Institute for Nanotechnology, Research Center Karlsruhe (P.W.), and the Danish Agency for Science, Technology and Innovation (J.P.).

*Author to whom correspondence should be addressed. koerting@tkm.physik.uni-karlsruhe.de

[1] I. Aleiner, P. Brouwer, and L. Glazman, Phys. Rep. 358, 309 (2002).

[2] W. G. van der Wiel et al., Rev. Mod. Phys. 75, 1 (2002).

[3] N. J. Craig et al., Science 304, 565 (2004).

[4] S. Sasaki et al., Phys. Rev. B 73, 161303(R) (2006).

[5] H. Jeong, A. M. Chang, and M. R. Melloch, Science 293, 2221 (2001).

[6] V.N. Golovach and D. Loss, Europhys. Lett. 62, 83 (2003).

[7] J. Simonin, Phys. Rev. Lett. 97, 266804 (2006).

[8] C. Jayaprakash et al., Phys. Rev. Lett. 47, 737 (1981); B. A. Jones and C. M. Varma, Phys. Rev. Lett. 58, 843 (1987); Phys. Rev. B 40, 324 (1989).

[9] G. Zarand et al., Phys. Rev. Lett. 97, 166802 (2006).

[10] C.-H. Chung and W. Hofstetter, arXiv:cond-mat/0607772.

[11] L. Zhu and C. M. Varma, arXiv:cond-mat/0607426.

[12] M. G. Vavilov and L. I. Glazman, Phys. Rev. Lett. 94, 086805 (2005).

[13] P. Simon, R. López, and Y. Oreg, Phys. Rev. Lett. 94, 086602 (2005).

[14] A. Kaminski, Yu. V. Nazarov, and L. I. Glazman, Phys. Rev. B 62, 8154 (2000).

[15] A. Rosch, J. Kroha, and P. Wölfle, Phys. Rev. Lett. 87, 156802 (2001).

[16] S. Sachdev and R. N. Bhatt, Phys. Rev. B 41, 9323 (1990).

[17] A. A. Abrikosov, Physics (N.Y.) 2, 5 (1965).

[18] O. Parcollet and C. Hooley, Phys. Rev. B 66, 085315 (2002); J. Paaske, A. Rosch, and P. Wölfle, Phys. Rev. B 69, 155330 (2004).

[19] A. Rosch, J. Paaske, J. Kroha, and P. Wölfle, Phys. Rev. Lett. 90, 076804 (2003); J. Phys. Soc. Jpn. 74, 118 (2005).

[20] J. Paaske et al., Phys. Rev. B 70, 155301 (2004); Nature Phys. 2, 460 (2006). 